\title{
ESTUDO DOS GEMINADOS DO TIPO BRASIL EM AMETISTA DA BAHIA ATRAVÉS DA TOPOGRAFIA DE RAIOS X E LUZ POLARIZADA
}

\author{
ZBIGNIEW BARAN* , SHIGUEMI FUJIMORI** e RILSON RODRIGUES DA SILVA***
}

\begin{abstract}
Brazil law type twinning is very common in quartz. Generally it is not observed in normal petrographic thin sections but it is possible to study this kind of twinning under polarized light in thicker sections. X-ray topography will be another powerfull method to study and it was applied for the amethyst of the Mina Cabeluda of the state of Bahia.
\end{abstract}

INTRODUÇÃo O quartzo é um dos minerais mais abundantes da crosta terrestre. Aparece em muitas rochas ígneas, metamórficas e sedimentares como uma das fases essenciais. Ocorre, xenomórfico ou idiomórfico, em variedades tais como quartzo hialino, quartzo róseo, ametista, quartzo esfumaçado e outras.

O quartzo é também opticamente ativo e pode rodar o plano de polarização da luz para a direita (quartzo dextrógiro) ou para a esquerda (quartzo levógiro). Esta propriedade é uma conseqüência da estrutura cristalina do mineral. $\mathrm{O}$ quartzo se cristaliza na classe 32 , cujo eixo ternário é helicoidal do tipo $3_{1}$ ou $3_{2}$, e disso resultam os dois grupos espaciais $\mathrm{P} 3_{1} 21$ e $\mathrm{P}_{2} 21$, correspondentes ao quartzo direito e esquerdo, respectivamente, e portanto enantiomorfos. Esses grupos espaciais possuem ainda três eixos de rotação binários, normais ao eixo ternário. Desse modo, o enantiomorfismo do quartzo é uma conseqüência do arranjo helicoidal dos tetraedros de $\mathrm{SiO}_{4}$ em torno do eixo ternário (Fig. 1), de forma dextrógira ou levógira.
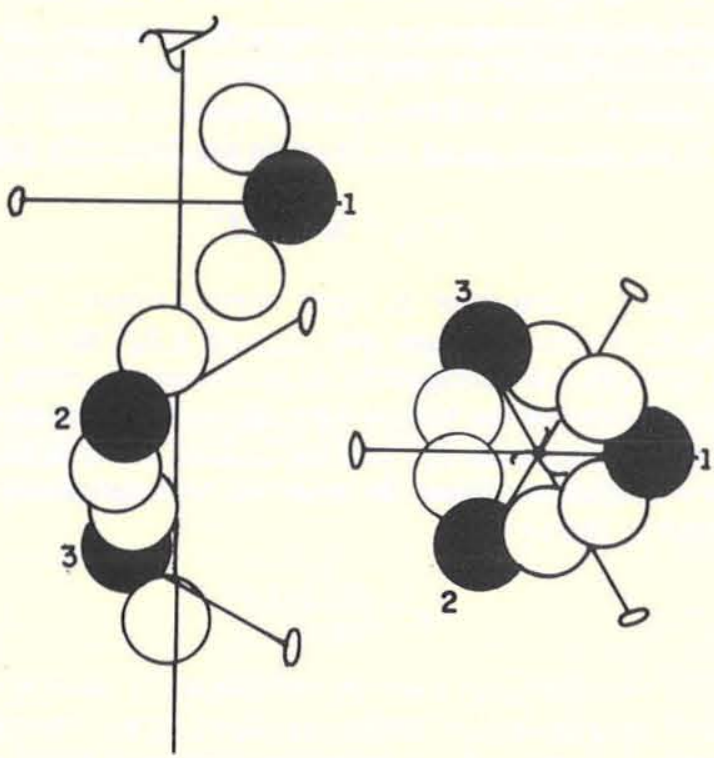

Figura 1 - Arranjo dos átomos de $\mathrm{Si}$ e $\mathrm{O}$ no quartzo em torno do eixo ternário
Uma outra característica do quartzo é sua geminação. Muitos tipos de geminação estão presentes, embora pouco observados nos estudos petrográficos, por causa da espessura da lâmina.

Na geminação do tipo Dauphiné, os indivíduos geminados são de quartzo direito ou esquerdo relacionados pela rotação de 1800 em torno do eixo cristalográfico $c$. Os eixos cristalográficos $c$ dos indivíduos são paralelos, mas a polaridade elétrica dos eixos $a$ é invertida, razão pela qual esse tipo de geminação é também denominada geminação elétrica. É uma geminação do tipo interpenetrante.

$\mathrm{Na}$ geminação do tipo Brasil, as partes de quartzo direito e quartzo esquerdo estão relacionadas pela reflexão nos planos $(11 \overline{2} 0)$. Os eixos cristalográficos são paralelos mas as polaridades elétricas dos eixos $a$ são também invertidas, o que torna a geminação freqüentemente do tipo polissintético.

Além dessas geminações, o quartzo mostra outros tipos como tipo Japão etc. (Frondel 1962).

A geminação tipo Brasil aparece freqüentemente na ametista na face romboédrica positiva $r$ (1011), mas os estudos detalhados dessa face usando o microscópio de luz polarizada e topografia de raios-X não tinham sido efetuados e apenas nos últimos anos eles foram iniciados (Baran et al. 1981 e 1986).

A ametista da Mina Cabeluda, na parte norte da Chapada Diamantina, no Estado da Bahia, apresenta-se geminada na face romboédrica e foi estudada com luz polarizada e por topografia de raios-X.

\section{ESTUDO COM LUZ POLARIZADA DA GEMINAÇÃO} BRASIL A identificação óptica dos geminados de quartzo é possível para a geminação do tipo Brasil em que coexistem os enantiomorfos esquerdo e direito, cada um girando o plano de polarização para a esquerda e direita, respectivamente. Numa lâmina delgada da seção basal do quartzo, de espessura padrão de $0,03 \mathrm{~mm}$, a rotação provocada pelo mineral é dà ordem de $0,65^{\circ}$ para radiação amarela de sódio, tornando o geminado praticamente imperceptível no microscópio petrográfico com nicóis cruzados.

Para a observação do geminado polissintético do tipo Brasil, uma fatia de $2 \mathrm{~mm}$ de espessura cortada paralelamente à face $r(1011)$ de um cristal de ametista da mina Cabeluda foi montada na platina universal Zeiss de quatro eixos. Como o eixo cristalográfico $c$ do quartzo se acha inclinado

\footnotetext{
* Instituto de Física da UFBa. Rua Caetano Moura, 123, Federação, CEP 40000, Salvador, Bahia, Brasil

** Instituto de Geociências da UFBa. Rua Caetano Moura, 123, Federação, CEP 40000, Salvador, Bahia, Brasil

*** Centro Tecnológico da UFPe. CEP 50000, Recife, Pernambuco, Brasil
} 
de cerca de $38^{\circ}$ em relação a essa face romboédrica, a rotação em torno dos eixos da platina permite trazê-lo à posição perpendicular à platina do microscópio, isto é, $c$ coincidente com o eixo principal do microscópio. Nesssa posição e com os nicóis cruzados, a geminação aparece na forma de várias lamelas (Foto 1) separadas por linhas escuras, que são os traços do plano de composição. A rotação do analisador para a esquerda extingue um conjunto de lamelas alternadas do quartzo e a rotação para outro lado extingue as demais lamelas do outro tipo (Fotos 2 e 3 ).

ESTUDO DOS GEMINADOS DO TIPO BRASIL ATRAVÉS DA TOPOGRAFIA DE RAIOS-X No quartzo geminado do tipo Brasil, as duas variedades esquerda e direita aparecem juntas, uma relacionada à outra por reflexão. Trata-se, portanto, do problema de distinguir entre a estrutura helicoidal levógira e dextrógira, chamada em cristalografia de configuração absoluta.

Para examinar essas duas estruturas por meio de difração de raios-X, é necessário considerar, respectivamente, os fatores da estrutura $F_{L}$ (hkil) e $F_{D}$ (hkil) das estruturas levógira e dextrógira. $\mathrm{O}$ fator de estrutura depende tanto da natureza dos átomos contidos na cela unitária, através dos respectivos fatores de espalhamento atômico, $f_{k}$, como da distribuição dos átomos na cela unitária, através de suas coordenadas $x k, y k, z k$.

Considerando-se a expressão do fator de estrutura para uma configuração levógira:

$$
F_{L}(h k i l)=\sum_{\mathrm{k}} f_{k} \exp \mathrm{i} 2 \pi\left(h x_{k}+k y_{k}+l z_{k}\right)
$$

em que $h, k$ e $l$ são os índices de Miller de uma família de planos reticulares, a configuração dextrógira, então, pode ser considerada como o resultado da inversão de todas as coordenadas da estrutura $L$ pela operação de reflexão através de um dos planos de forma $(11 \overline{2} 0)$. $O$ operador de reflexão transforma um ponto $P(x y z)$ em ponto $P^{\prime}(\bar{x} \bar{y} z)$ e, em seguida, o operador do eixo binário, que é o eixo perpendicular ao plano de reflexão $(11 \overline{2} 0)$, transforma o ponto $P^{\prime}(\bar{x} \bar{y} z)$ em ponto $P^{\prime \prime}(\bar{x} \bar{y} \bar{z})$. Assim, a cada ponto $(x y z)$ de $L$ corresponde um ponto simétrico $(\bar{x} \bar{y} z)$ de $D$, de modo que o fator $F_{D}$ é dado por:

$$
F_{D}(h k i l)=\sum_{\mathrm{k}} f_{k} \exp -i 2 \pi\left(h x_{k}+k y_{k}+1 \mathrm{z}_{k}\right)=F_{L}{ }^{*}
$$

em que $F_{L}$ ", é o complexo conjugado de $F_{L}$. Como a intensidade do feixe difratado $I$ (hkil) é proporcional ao produto do fator de estrutura por seu complexo conjugado, $I($ hkil $)=$ $F\left(\right.$ hkil). $F^{*}$ (hkil), têm-se $I_{L}=F_{L} \cdot F_{L}{ }^{*}=F_{D}{ }^{*} \cdot F_{D}=I_{D}$. E impossível pelos métodos clássicos de difração dos raios $\mathrm{X}$ determinar a configuração absoluta de uma estrutura helicoidal (enantiomorfa). Isto é uma conseqüência da lei de Friedel, a qual diz serem as intensidades de uma reflexão $I(h k i l)$ e da outra anti-reflexão $I(\bar{h} \bar{k} i l)$ iguais ou, em outros termos, exibir o padrão de difração de raios-X sempre um centro de simetria, mesmo que a estrutura que produz este padrão não o possua (Friedel 1913).

Esta particularidade não permite, à primeira vista, reconhecer a configuração de uma estrutura enantiomorfa. Um par enantiomorfo dá exatamente o mesmo padrão de difração, pois uma estrútura dela se transforma em outra por inversão de todas as coordenadas.

Sabe-se, desde as experiências' de Coster et al. (1930), que esta lei de Friedel não tem aplicação universal e que, em certas circunstâncias, é possível determinar mesmo as configurações das estruturas enantiomorfas.

Princípio do Método A difração de raios-X por um cristal é um resultado do espalhamento coerente por elétrons dos átomos contidos na estrutura do cristal. Considera-se o espalhamento por um elétron como uma reemissão da radiação por um oscilador-elétron posto em vibrações forçada pelo campo elétrico da onda dos raios-X incidentes. Assim, pode ser formulada a equação dinâmina do movimento oscilador e feita uma análise em função da freqüência própria do elétron $\omega_{0}$, e a freqüência da radiação incidente $\omega$. Para os métodos clássicos de difração, a freqüência da radiação incidente $\omega$ é muito maior que a freqüência própria do elétron do átomo, $\omega_{0},\left(\omega_{0}<<\omega\right)$ (isto é, o comprimento de onda de raios-X, $\lambda=\frac{2 \pi c}{\omega}$, é muito menor que os comprimentos de onda da radiação $\mathrm{X}$ correspondente à aresta de absorção significando um espalhamento normal). Quando o comprimento dos raios-X incidentes se aproximar do comprimento de onda correspondente à aresta de absorção $\left(\lambda=\lambda_{0}=\frac{2 \pi \mathrm{c}}{\omega_{0}}\right)$, resulta um espalhamento anômalo chamado de efeito de dispersão anômala na teoria da difração dos raios-X. O tratamento formal da dispersão anômala na teoria da difração de raios-X é já bem conhecido e uma boa revisão é apresentada por James (1958).

Assim é possível, pelo menos em princípio, adaptar a freqüência da onda incidente de tal maneira que se atinja a ressonância para os elétrons $\mathrm{K}$ de um átomo que compõe um cristal. Estes elétrons dariam à onda resultante difratada pelo átomo uma defasagem anômala diferente de 0 ou de $\pi$ em relação à onda incidente.

Quando o comprimento de onda $\lambda$ dos raios- $\mathrm{X}$ incidentes sobre um átomo contido no cristal se aproximar do comprimento de onda $\lambda o$, correspondente à sua aresta de absorção, o fator de espalhamento atômico torna-se complexo como conseqüência matemática da equação dinâmica do movimento oscilatório do elétron causado pela onda incidente de raios-X. Isto se traduz matematicamente dando a seguinte expressão complexa ao fator de espalhamento atômico:

$$
f=f_{o}+\Delta f^{\prime}+i \Delta f^{\prime \prime}
$$

em que $f_{\mathrm{o}}$ é um fator de espalhamento atômico "normal" (isto é, $\omega$ é muito maior que $\omega_{0}$ ); $\Delta f^{\prime}$ e $\Delta f$ " são os termos da correção real e imaginária, respectivamente, cujos valores podem obter-se nas tabelas internacionais de cristalografia. O termo imaginário $\Delta f^{\prime \prime}$ implica a absorção verdadeira, sendo o coeficiente linear de absorção correspondente a um átomo $k$, dado por:

$$
\mu_{a}=\frac{2 \mathrm{e}^{2} \lambda}{m c^{2}} \Delta f^{\prime \prime}
$$

Assim sendo, por meio do espalhamento anômalo introduz-se no padrão de difração um elemento que caracteriza o próprio átomo e não somente sua posição entre os outros. Os fatores de espalhamento atômico complexos não só diferem pelo sinal de suas partes imaginárias mas também por seus valores absolutos, podendo isto ser verificado pelo cálculo dos fatores de estrutura.

Para a estrutura enantiomorfa $L$, o fator de estrutura e seu complexo conjugado podem ser escritos numa ex- 

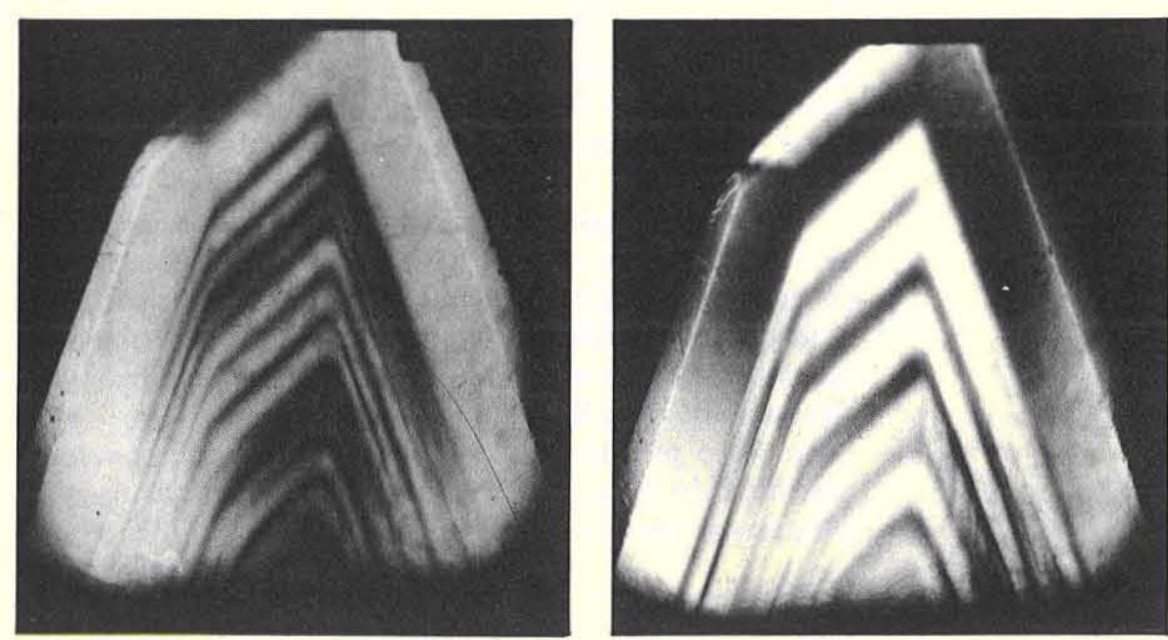

Foto 1 - Contatos entre lamelas
Foto 2 - Lamelas dextrógiras extintas

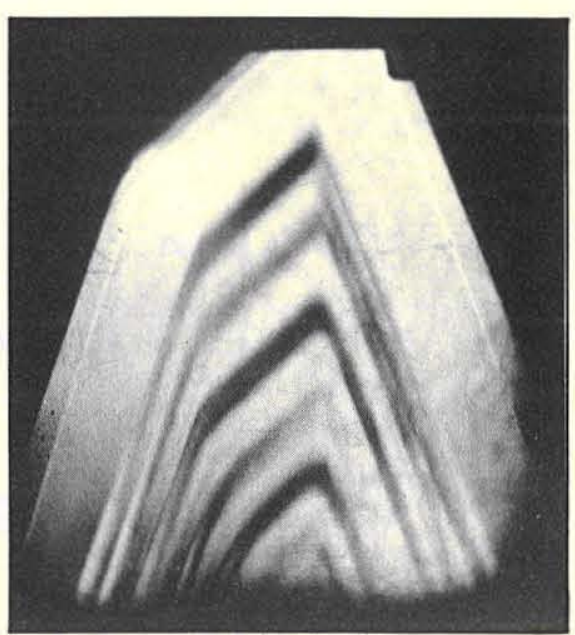

Foto 3 - Lamelas levógiras extintas pressão simples, juntando no fator de espalhamento atômico, $f\left(f=f_{0}+\Delta f^{\prime}+i \Delta f^{\prime \prime}\right)$ a parte real em um termo $r$, resultando $f=r+i$ sk e assim:

$$
\begin{array}{r}
F_{L}^{\prime}(h k l)=\sum_{k}\left(r_{k}+i s_{k}\right) \exp i 2 \pi\left(h x_{k}+k y_{k}+l z_{k}\right) \\
F_{L}^{\prime *}(h k l)=\sum_{k}\left(r_{k}-i s_{k}\right) \exp -i 2 \pi\left(h x_{k}+k y_{k}+l z_{k}\right) .
\end{array}
$$

No espalhamento normal, $s_{k}$ são iguais a zero, exceto para um átomo cujos elétrons $K$ estejam em ressonância com a radiação $X$ incidente.

De um modo análogo, temos o mesmo para a estrutura enantiomorfa D:

$$
F_{D}^{\prime}(h k l)=\sum_{k}\left(r_{k}-i s_{k}\right) \exp -\mathrm{i} 2 \pi\left(h x_{k}+k y_{k}+i z_{k}\right)
$$

Esta expressão não é mais igual ao complexo conjugado $F_{L}^{\prime}$, donde $I_{L}^{\prime}(h k l) \neq I_{D}^{\prime}(h k l)$.

A lei de Friedel também não é mais válida, pois pode-se verificar facilmente que $I_{L}^{\prime}(h k l) \neq I_{L}^{\prime}(\bar{h} \bar{k} l)$, isto é, as intensidades de reflexão $h k l$ e da anti-reflexão $(\hbar k l)$ não são iguais.

Uma das mais interessantes aplicações do espalhamento anômalo foi a determinação da configuração absoluta do quartzo- $\alpha$ (Vries 1958).

As medidas das intensidades de certas reflexões de Vries foram aproveitadas e aplicadas para determinar os geminados em ametista através da técnica de topografia de raios-X.

Procedimento Experimental No cristal de quartzo, do ponto de vista prático, o átomo de $\mathrm{Si}$, cujo número atômico $Z$ é maior que o de $\mathrm{O}$, está predisposto a espalhar os raios-X de maneira anômala. $\mathrm{O}$ átomo de $\mathrm{Si}$ possui a aresta de $\mathrm{ab}$ sorção para os elétrons $K$ igual a $\lambda_{0}=6,7446 \AA$, enquanto que, para o átomo de 0 , este valor é $\lambda_{0}=23,32 \AA$.

Embora não podendo realizar as condições de ressonância entre a freqüência própria dos elétrons do átomo de $\mathrm{Si}$ e a freqüência da onda incidente $\left(\omega=\omega_{0}\right.$ ou $\left.\lambda_{0}=\frac{2 \pi c}{\omega_{0}}\right)$, pode-se aproximar à aresta de absorção de modo que a par- te imaginária do fator de espalhamento atômico $f=$ $f_{o}+\Delta f^{\prime}+i \Delta f^{\prime \prime}=r+i s$ para o átomo de Si não seja desprezível. Isto é realizável utilizando a radiação $\mathrm{K}_{\alpha}$ do $\mathrm{Cr}$, cujo comprimento de onda é $\lambda=2,28962 \AA\left(\omega_{0}<\omega\right)$.

Para poder realizar a experiência escolheu-se o plano $(1 \overline{2} 11)$ em um indivíduo geminado e o plano (1121) no outro indivíduo. Quando a lei de Friedel é obedecida, as intensidades das reflexões $1 \overline{2} 11$ e $\overline{1121}$ são iguais. Isto vem do fato de estas reflexões serem estruturalmente equivalentes pela operação de reflexão através de um dos planos da forma (1120). Quando o espalhamento é anômalo, a diferença de intensidade entre essas reflexões é igual a 43\% para a radiação $\mathrm{K} \alpha$ do $\mathrm{Cr}$ (Vries 1958).

A identificação das lamelas levógiras e dextrógiras foi efetuada pela técnica de topografia de raios-X, por reflexão, cujo princípio está esquematizado na figura 2, chamada também de topografia de Berg-Barrett (Austerman \& Newkirk 1967).

$\mathrm{O}$ feixe incidente emerge de uma fenda divergente e incide corretamente sobre uma face do cristal estudado. $\mathrm{O}$ cristal é colocado de modo que se obtém um feixe difratado por uma família predeterminada de planos, os quais não são paralelos às faces do cristal (reflexão assimétrica). 0 ângulo entre o feixe incidente e a face do cristal é ajustado sendo que somente a radiação característica $\mathrm{K}_{\alpha}$ do tubo de raios-X é difratada pela família de planos cujo vetor recíproco é indicado por $\overrightarrow{\mathrm{g}}_{h k l}$. O feixe difratado é limitado por uma segunda fenda, depois é registrado num filme colocado perpendicularmente ao mesmo. Se o filme e o cristal são relacionados e animados de um movimento de translação, obtém-se uma fotografia do conjunto de face do cristal, isto é, topografia em translação.

A topografia de raios-X por reflexão produz uma imagem da superfície do cristal através do registro das intensidades dos raios-X difratados por esta superfície.

Existem dois parâmetros que determinam o nível de intensidade atingido em cada ponto do filme, isto é, o contraste da imagem de difração (Authier 1977). O primeiro parâmetro simplesmente determina uma situação em que 


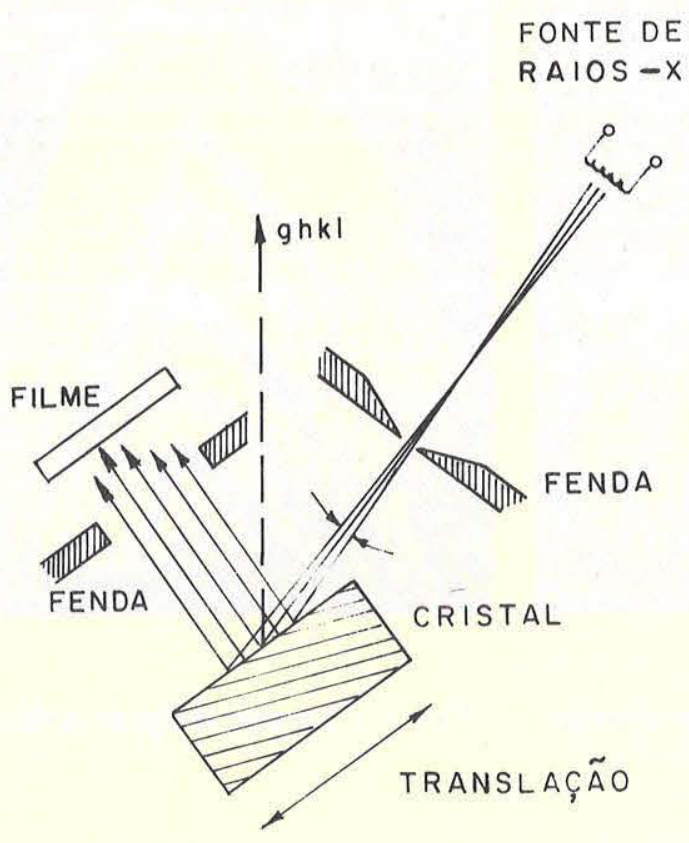

Figura 2 - Esquema gráfico da técnica de topografia de raios-X por reflexão

estão duas regiões desorientadas no cristal, uma em relação à outra e que podem estar ou não orientadas de modo a refletir os raios-X de acordo com a lei de Bragg. A intensidade desses raios refletidos é variável. Esta variação em intensidade de ponto a ponto na imagem de difração chama-se de contraste de orientação (ou desorientação) e fornece uma medida da desorientação entre as partes cristalinas da rede cristalina.

O segundo parâmetro, que determina a intensidade, é a perfeição da rede de cada cristal.

Os fatores físicos, como o comprimento de onda de raios-X, absorção do cristal e o fator de espalhamento atômico para uma reflexão de Bragg, determinam o nível de intensidade dos feixes difratados, podendo estar entre dois casos extremos correspondentes à região do cristal idealmente perfeito e à região do cristal idealmente imperfeito. $\mathrm{O}$ contraste proveniente da variação na perfeição da rede de ponto a ponto é convenientemente chamado de contraste de extinção. Este contraste é considerado como o efeito da extinção primária consistindo na diminuição da intensidade dos feixes difratados num cristal perfeito, como resultado das reflexões múltiplas produzidas dentro do cristal durante a difração (Fig. 3). Por exemplo, um feixe refletido duas vezes no cristal tem a mesma direção que o feixe incidente, mas, devido à mudança da fase de $\pi / 2$ durante cada reflexão, estará ele em fase oposta ao feixe incidente e assim resultando um decréscimo na intensidade, isto é, extinção parcial, como resultado da interferência destrutiva desses feixes. Essas reflexões múltiplas não ocorrem nas regiões do cristal, onde a periodicidade da rede é perturbada. A presença dos defeitos dá lugar à perturbação na propagação dos feixes de raios-X.

A topografia de raios-X permite revelar os defeitos na rede cristalina pelos efeitos introduzidos por eles no cristal perfeito que os circunda: um campo de deformação no caso geral ou uma translação, ou uma rotação de uma parte da rede cristalina em relação a outra, por conseqüência, o contraste é necessariamente devido ao efeito de desorientação ou ao efeito de extinção.

Um defeito como, por exemplo, um deslocamento ou uma inclusão introduz as deformações na rede cristalina. Outro tipo de defeito é o de superfície, que é uma descontinuidade da superfície separando as duas regiões perfeitas ou quase perfeitas no cristal, e portanto dilacera a propagação das ondas de raios-X.

Isto é o presente caso de geminação em que os valores absolutos do fator de estrutura são diferentes nas duas regiões dos geminados $\left(F_{L}\right.$ e $\left.F_{D}\right)$ (Kato 1974 e 1975).

RESULTADOS EXPERIMENTAIS E DISCUSSÃO Empregando a topografia de raios-X por reflexão e a radiação $\mathrm{K} \alpha$ do $\mathrm{Cr}$ foram obtidas as topografias do plano (1121) apresentadas na foto 4 e do plano (1211) na foto 5. $\mathrm{O}$ ângulo de Bragg para esta radiação é $\theta_{B}=30,8^{\circ}$ e o ângulo entre o plano de reflexão e a face romboédrica (1011) do cristal estudado é igual a $28^{\circ}$. As topografias foram registradas nas placas fotográficas Ilford G5 nuclear plates.

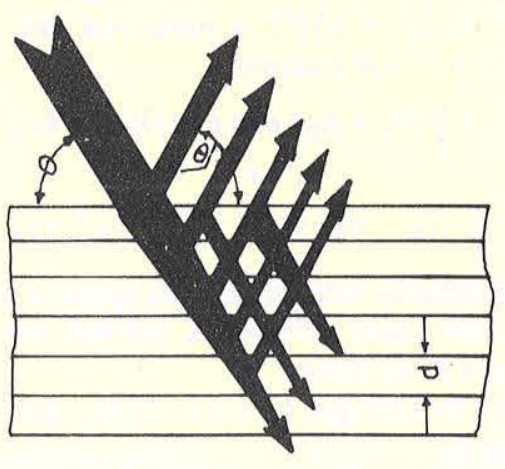

Figura 3 - Extinção primária dos raios-X num cristal perfeito

Observou-se nas topografias uma seqüência alternada de bandas brancas e escuras. As bandas brancas da topografia para o plano (11121) (Foto 4) correspondem às bandas escuras da topografia para o plano (12 111 ) (Foto 5), significando nitidamente que as intensidades $I_{(\overline{1121})}$ e $I_{(1 \overline{2} 11)}$ não são iguais. Sabendo-se que, neste par de reflexões, a 1121 é equivalente à anti-reflexão (1211), a diferença entre suas intensidades é causada pelo efeito da dispersão anômala. Conseguiu-se assim diferenciar as lamelas dos geminados levógiros e dextrógiros arranjados alternadamente na face da ametista estudada.

A topografia (Foto 6) feita nas mesmas condições experimentais, mas com radiação $\mathrm{K} \alpha$ do $\mathrm{Cu}$, apresenta somente as linhas escuras identificadas pela fotomicrografia (Foto 1) como sendo contatos entre as lamelas de geminados, levógiras e dextrógiras, não apresentando um contraste visível entre as lamelas.

Nas fotomicrografias em luz polarizada, representadas nas fotos 2 e 3 , aparece a mesma seqüência alternada de 


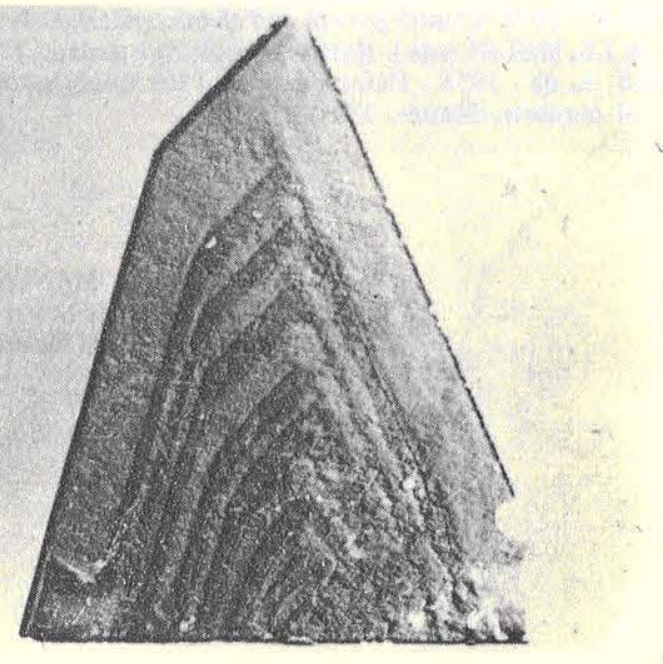

Foto 4 - Lamelas dextrógiras paralelas a (1121) extintas usando radiação $K_{\alpha}$ do $\mathrm{Cr}$

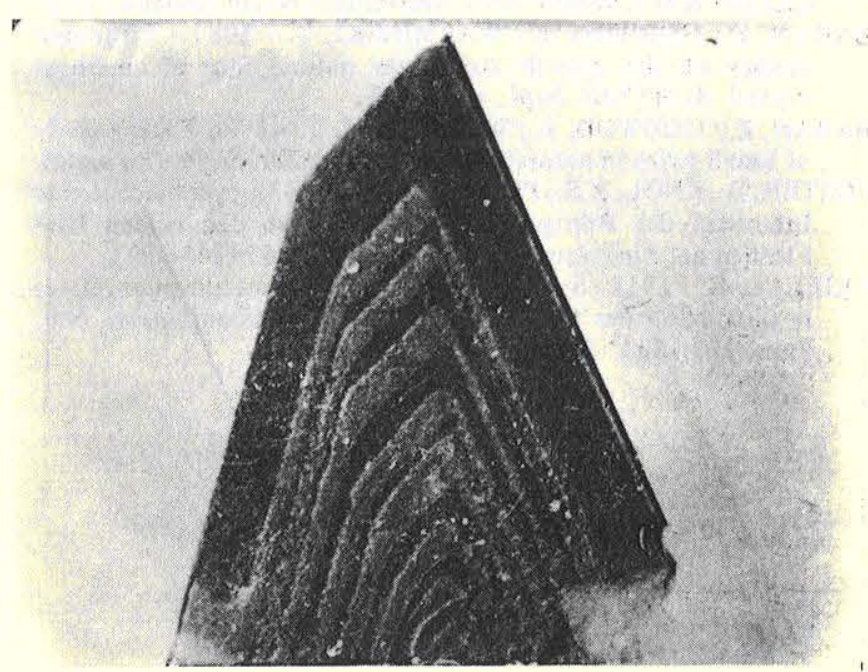

Foto 5 - Lamelas levógiras paralelas a (1211) extintas usando a radiação $K_{\alpha}$ do $\mathrm{Cr}$

faixas brancas e escuras, correspondendo às lamelas levógiras e dextrógiras.

As lamelas levógiras e dextrógiras extintas alternadamente no microscópio de polarização correspondem às mesmas lamelas, respectivamente, das topografias de raios-X, empregando o efeito da dispersão anômala.

Os resultados aqui apresentados confirmam inequivocamente a presença de geminados repetidos ou polissintéticos do tipo Brasil na face natural romboédrica da ametista, até hoje não evidenciado.

CONCLUSÕES Neste trabalho foi demonstrada, pela primeira vez, a utilidade do método de topografia de raios-X, empregando o efeito da dispersão anômala na diferenciação dos geminados do tipo Brasil no quartzo, usado em conjunto com a microscopia de luz polarizada.

É um método alternativo de investigação dos geminados do tipo Brasil.

A fim de se obter um conhecimento mais completo sobre este tipo de geminados é necessário estudá-lo pela topografia por transmissão. Este método permite caracterizar as lamelas de geminação contidas no interior de uma placa do cristal de $2 \mathrm{~mm}$ de espessura aproximadamente. Neste caso é indispensável empregar a radiação de síncrotron devido à al ta absorção da radiação $\lambda=2,29 \AA$ pela ametista.

Agradecimentos Os autores agradecem ao Conselho Nacional do Desenvolvimento Científico e Tecnológico pelas bolsas de estudo concedidas; à dona Eraldina Leão Campêlo, pela datilografia; e a Warney Bispo de Magalhães, pela confecção dos desenhos. 


\section{REFERÊNCIAS BIBLIOGRÁFICAS}

AULEYTNER, J. - 1967 - X-ray Methods in the study of defects in single crystals, Warszawa. Pergamon Press, Polish Scientific Publishers.

AUSTERMAN, S.B. \& NEWKIRK - 1967 - Experimental procedures in X-ray diffraction topography, Advan. X-ray Anal., 10:134 Plenum Press, N. York.

AUTHIER, A. - 1977 - Crystal Growth and Materials. In: E. KALDIS \& H.J. SCHEL eds.), Amsterdam, North-Holland $516 \mathrm{p}$

BARAN, Z.; GODWOD, K.; WARMINSKI, T. - 1981 - Structural studies of the growth stripes on natural face of amethyst crystal. Acta Cryst. Supl., a37:c-143.

BARAN, Z.; GODWOD, K.; WARMINSKI, T. - 1986 - X-ray study of Brazil twins in natural amethyst. Phys. Sat. Solid. (no prelo)

COSTER, D.; KNOL, K.S.; PRINS, S.A. - 1903 - Unterschiede in der Intensität der Röntgenstrahlenreflexion an den beiden III Flächen der Zinkblende. Z. Physik, Berlim, 63:(345-369).

FRIEDEL, G. - 1913 - Sur les symétries crystallines que peut réléver la diffraction des rayons Rontgen. Compt. Rend. Acad. Sci., Paris, 157:1533.
FRONDEL, C. - 1962 - The Dana's system of Minerallogy, 7th ed. N. York John Wiley \& Sons, v. 3.

JAMES, R.W. - 1958 - The optical principles of diffraction of $X$-rays. London. Bell and Sons.

International Tables for X-Ray Crystallography, Birminghan (1969-1974) Kynoch Press, v. 3.

KATO, N. - 1974 - X-ray diffraction. In: AZAROFF, L.V. et al. (eds.). N.Y., McGraw-Hill, 222 p.

KATO, N. - 1975 - Crystal growth and characterization. In: R. UEDA, \& J.B. MULIN (eds.). North-Holland; Amsterdan, 279 p.

VRIES, A. de - 1958 - Determination of the absolute configuration of $\alpha$-quartz. Nature, 181:1193.

MANUSCRITO 394

Recebido em 10 de Setembro de 1986 Revisão aceita em 03 de Fevereiro de 1987

... “Um geólogo sem imaginação é tão ineficiente quanto um pato sem as membranas natatórias entre os dedos"... 